\title{
Preparation and Application of Anionic and Cationic Waterborne Polyurethanes and Graphene-Cellulose Nanocrystal as an Antistatic Agent for Cashmere
}

\author{
Mingxing Yang1\#, Khusniddin Ismoilov#, Sachin Chauhan11, Quan Heng1*, Zulaykho Islamova² \\ ${ }^{1}$ Chemistry and Chemical Engineering, Wuhan Textile University, Jiangxia District, Wuhan, Hubei, China \\ ${ }^{2}$ Chemical Technology, Tashkent Institute of Textile and Light Industry, Yakkasaroy District, Tashkent, Uzbekistan \\ Email: husmaf9396husmaf9396@gmail.com, sachinch20.94@gmail.com, zulayho.islamova@mail.ru, *quanheng2002@163.com
}

How to cite this paper: Yang, M.X., Ismoilov, K., Chauhan, S., Heng, Q. and Islamova, Z. (2019) Preparation and Application of Anionic and Cationic Waterborne Polyurethanes and Graphene-Cellulose Nanocrystal as an Antistatic Agent for Cashmere. Graphene, 8, 19-31.

https://doi.org/10.4236/graphene.2019.82002

Received: April 14, 2019

Accepted: April 27, 2019

Published: April 30, 2019

Copyright ( 2019 by author(s) and Scientific Research Publishing Inc. This work is licensed under the Creative Commons Attribution International License (CC BY 4.0).

http://creativecommons.org/licenses/by/4.0/

cc) (i) Open Access

\begin{abstract}
The main purpose of this research work is to improve anti-static properties of Cashmere fabric by introducing application comprising anti-static agent by foaming which was made with cationic waterborne polyurethane and graphene-CNC. Cashmere fabric was cut into 10 pieces of sample cloth of $5 \mathrm{~cm}$ * $5 \mathrm{~cm}$ size, washed with acetone solution, and then dried in an oven at $60{ }^{\circ} \mathrm{C}$. Three forms of waterborne polyurethanes such as two forms of Cationic waterborne polyurethane (CWPU) and a form of Anionic waterborne polyurethane (AWPU) were synthesized. Cellulose nanocrystalline (CNC)/graphite powder solution with the ratio of $0.5 / 1,1 / 1,2 / 1$ was prepared by ultrasonic probe stripping method, and the concentration of graphite powder was ensured to be $1 \mathrm{mg} / \mathrm{ml}$. The fabric was treated with anionic and cationic WPUs foaming solution until the weight gain reached 2.5 - $3.5 \mathrm{wt} \%$. After drying, the elastic cloth was foamed with graphene solution until the graphite content of the cloth was close to $10 \%, 20 \%, 40 \%, 60 \%$ respectively, and then dried for reserving. Characterization properties of pure graphite powder, pure CNC and graphene solution with different proportions of three components were tested by Fourier transform infrared spectrometer (FTIR), X-ray diffraction (XRD), Thermalgravitimetric analysis (TGA) and scanning electron microscopy (SEM). Take the original cloth, only WPU treated cloth and four clothes with different graphite content for the fabric performance test.
\end{abstract}

\section{Keywords}

Cashmere Fabric, Anionic and Cationic Waterborne Polyurethane, Graphene, Cellulose Nanocrystal, Anti-Static Property 


\section{Introduction}

The utilization of traditional solvent that is based on Polyurethane (PU) has regularly been being deterred, because of firm environmental and governmental influences [1]. Textiles and other spheres such as adhesives, coatings, construction materials, inks as well as biological materials could be fields in which Waterborne polyurethane (WPU) might be utilized as a kind of multifunctional environmentally friendly substance due to its excellent properties. In terms of the outlook of material technology, the electrical properties, acoustic, optical properties as well as static and dynamic mechanical features are considered as essential properties and the performances of PU plastic are chemical, biological and corrosion resistance, adhesive properties. In spite of being a substantial number of excellent advantages of WPU, there might be found several disadvantages of WPUs which were recorded and published in some researches. As an example, it can be shown that if compared to a traditional solvent which is based on polyurethanes, a feebler drying rate is observed on WPUs because of the high secretive heat and evaporation of water. Furthermore, the linear hydrophilic groups that WPUs contain in their chain instigate to require too much drying time and higher energy utilization for WPUs to reach expected mechanical power. In the case of meeting the time efficiency and economic profit requirements, this could not be useful [2].

Hence, research nowadays is mainly focused on the preparation of WPUs with a high solid content, which is of growing interest both in the field of scientific research and in industrial applications [3]-[7]. As an important category of WPUs, cationic waterborne polyurethanes show excellent adhesion to anionic substrates, such as glass and leather [8]. For that reason, Cashmere is a soft wool fabric woven from the hair of the Cashmere which can be applied to an anionic substrate. Cashmere fibers are extremely soft and silky to the touch and weigh practically nothing. When even compared to Merino, Cashmere can be found softer and fine-drawn, and it is more delicate than other types of animal wool. Besides, Cashmere is especially useful in terms of using it for accessories and curtains. In many cases of specifications of wool fabrics, Cashmere can be admitted as the king of the fiber and treasure, owing to its pros and cons [9]. When comes to advantages of Cashmere, it can be counted that increasing the deep sleep which illustrates in experiments that cashmere material could enhance about more than 25 percentage of the individuals deep sleep time as well it can maintain heart rate slowly and steady. Additionally, moisture absorption and it keeps dry, warm in cold weather since cashmere special molecule structure can absorb the water vapor more than $35 \%$ into the hollow structure which cannot feel wettability and can remove quickly. Also, it presents no less than $40 \%$ natural elastic, after pressing, it has been observed that it could recover approximately $95 \%$ of initial ply. Moreover, as advantages of cashmere, fluffy, softness, natural fire-resistant, dust preventive are considered and cashmere has 17 kinds of abundant amino acid which provide the cashmere the feature of closing to the skin. Although here several pros have been mentioned, there some cons of cash- 
mere which are caused problems to fabricate products form it because cashmere is wool, it is a hydrophilic fiber, and wool fabrics frequently are highly charged caused by the firm friction between the wool scales. To solve this issue of cashmere fabric, anti-static finishing is required [10]. The importance of optimal performance is uniform fabric penetration while applying anti-static finishes to fabrics. In the finish formulation, the utilization of wetting agents is recommended, Cationic anti-static agents fit for exhaust processes [11]. The low add-on values (mostly about one or a few percents on the weight of fiber) enable spray and foam applications. Padding leads to lesser effects than foam does application with an identical add-on. The higher concentrations of anti-static agents on the fiber surface by the foam application can be the actual factor for this. Some of the potential side effects of the use of anti-static finishes include wearing comfort (no clinging and pleasant skin contact caused by hydrophilicity). To improve the anti-static ability of Cashmere fabric, graphene has been widely used over the last decade due to possessing excellent thermal, mechanical and electrical properties and high surface area. Graphene, a primary constructing block of graphite, possessing a one-atom-thick sheet of hexagonally comprised sp2-hybridized carbon atoms [12]. There have a lamellar structure and stacked jointly by van der Waals forces in the graphitic layers. Extraordinary electronic properties were anticipated to have in these individual layers. Graphene has extraordinary electrical and thermal conductivities, ultra-high theoretic specific surface area $\left(2630 \mathrm{~m}^{2} / \mathrm{g}\right)$ and high mechanical strength. These excellent properties make graphene a promising candidate for energy storage devices, composites, and electronics [13]. High specific surface area and electrical conductivity with mechanical strength make this material a promising choice for energy storage devices. For those aspects, some researchers have investigated experiments regarding graphene based on cellulose nanocrystal (CNC) which has hydroxyl group $(\mathrm{OH})$ presenting hydrophilicity as to improve electrical conductivity. Anti-static agents can be prepared by making of three forms of WPUs such as an anionic waterborne polyurethane and two cationic waterborne polyurethanes and graphene-based on cellulose nanocrystal (CNC) to apply that solution to coat on the surface of Cashmere fabric through a foam finishing. Here, the duty of WPUs is to serve as adhesives and to cover the graphene microparticles so that graphene microparticles are durable for several times laundarying, simultaneously WPUs can reduce pilling properties of Cashmere which is the most undesired.

The cost was high for the moisture evaporation of chemical reagents in the conventional fabric, finishing, however, the foam dyeing and finishing technology can save more energy in the drying process. Foam finishing is a kind of dying and finishing technology with great energy-saving effect because foam as a medium carrier of dyes was applied to substitute for part of the water. The processing effect can reduce $65 \%-75 \%$ compared with the conventional water consumption. Besides, multifunctional textiles can be produced through the foam finishing technology to meet the higher demand for market consump- 
tion. It is a good prospect for the application that the foam finishing technology is applied to Cashmere fabric finishing, including anti-pilling and anti-static [14][18].

In this current study, anti-static properties of Cashmere fabric was enhanced by casting film solution, which was prepared by mixing three types of WPUs and graphene-CNC with some concentrations of ratio for each sample, on the surface of it. The results obtained from tests showed that the anti-static properties of fabric were improved when compared with examples.

\section{Experimental}

\subsection{Materials}

Cashmere was purchased from Beta Textiles Co., Limited (BTEXCO). Isophorone diisocyanate (IPDI) and Methyl diethanolamine (MDEA), dibutyltin dilaurate (DBTDL), $\gamma$-Aminopropyl Triethoxysilane (KH550) chemical pure were obtained from Shanghai Jingchun Reagent Co., Ltd., and Aladdin Reagent (Shanghai) Co., Ltd; polyether triol (ZC330) industrial products Guangzhou Jinwang Chemical Co., Ltd.; N220, DMPA, Ethylenediamine (EA), Triethylamine (TEA) analytical purity China Pharmaceutical Group Chemical Reagent Co., Ltd. Graphene-CNC was derived by reaction of Graphite and cellulose nanocrystal withholding cold water temperature and centrifugal force to remove particles' residues. Anionic and cationic waterborne polyurethanes were synthesized by reacting reagents following their recipe. In synthesizing PTMG and PEG were dried and degassed under $110^{\circ} \mathrm{C}$ and other materials were used as received.

\subsection{Preparation of Cationic Waterborne Polyurethanes with MDEA Volume Ratio $20 \%$ and $13 \%$}

Two types of cationic waterborne polyurethanes were synthesized by following recipes shown in Table 1 and Table 2 as well as the chemical structure of Cationic Waterborne Polyurethanes was shown in Scheme 1.

The first step in the synthesis of the cationic WPUs was to introduce PTMG 2000 and 3667 which were vacuumed to remove water in them under $110^{\circ} \mathrm{C}$ and $0.01 \mathrm{Mpa}$ for 120 minutes with Isophorone diisocyanate (IDPI) which has functional group isocyanate $\mathrm{R}-\mathrm{N}=\mathrm{C}=\mathrm{O}$ which can react with chemicals when it is effected as a second step is done under $85^{\circ} \mathrm{C}$ for 150 minutes. The first prepolymer I was synthesized based on IDPI and PTMG. Then MDEA was reacted to build the second prepolymer for the final product to make it ready. A $250 \mathrm{~mL}$ round-bottomed, four-necked flask equipped with a nitrogen inlet, a condenser with a drying tube, a mechanical stirrer and a thermometer was used for the reaction. After being kept two chemicals at the required time, a drop of DBTDL was added as a catalyst and simultaneously by repeating six times of adding, MDEA was dropped at $40^{\circ} \mathrm{C}$ during the 30 minutes, then with the same repetition and the duration of time, NEP was added at $40^{\circ} \mathrm{C}-50^{\circ} \mathrm{C}$. In the next step, 


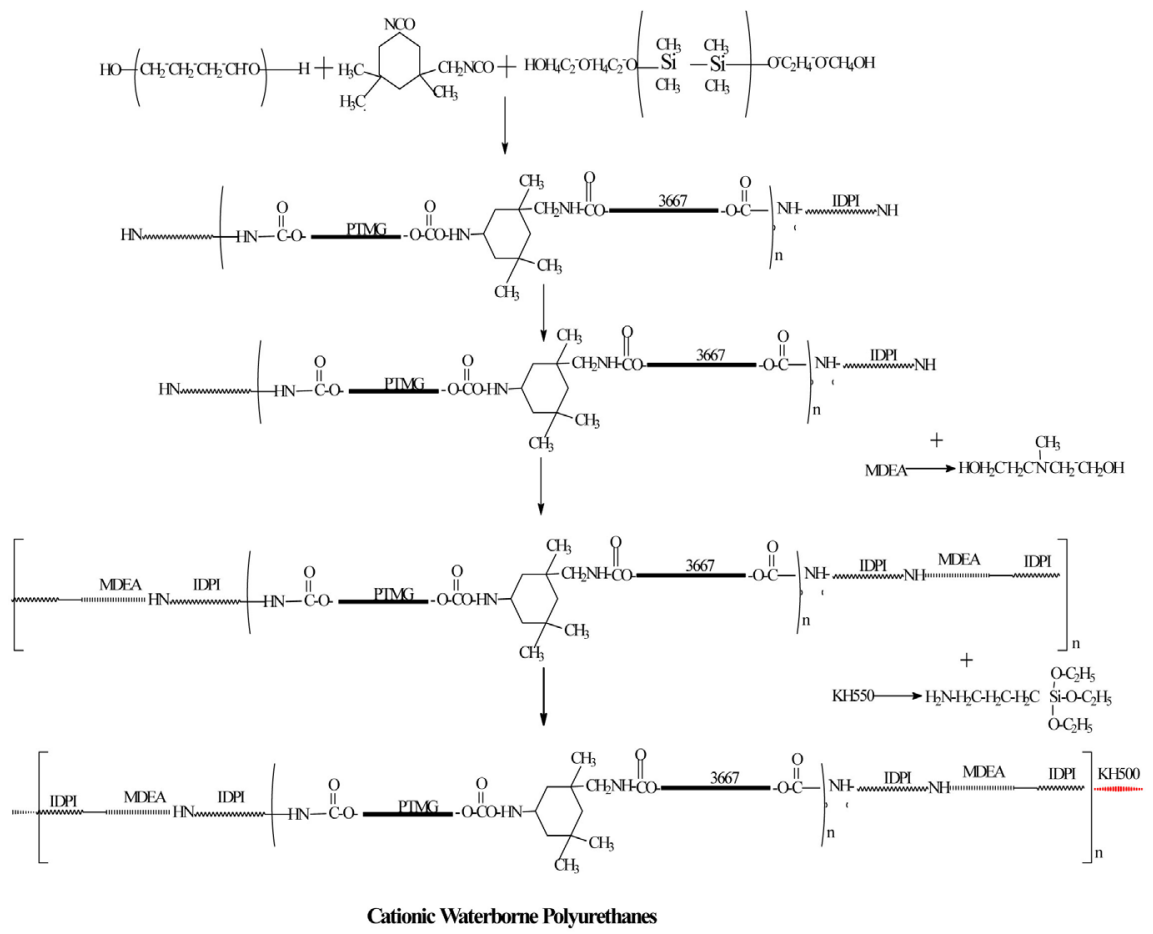

Scheme 1. The chemical structure of Cationic Waterbonre polyurethanes.

Table 1. CationWPU (MEDA Mass ratio 20\%).

\begin{tabular}{ccccc}
\hline Raw material name & mol & g & Mass ratio \% & Quantity \\
\hline PTMG & 0.07 & 140 & $13.30 \%$ & 11.67 \\
3667 & 0.07 & 154 & 44.34 & 12.83 \\
IDPI & 2.1 & 466.81 & 44.34 & 38.90 \\
DBTL & & & 1 & \\
MDEA & 1.8 & 214.49 & 20.37 & 17.87 \\
KH550 & 0.35 & 77.49 & 7.36 & 6.46 \\
Bromabutane & 1.8 & 246.64 & & 20.55 \\
Acetic acid & & & & 21.45 \\
\hline
\end{tabular}

Table 2. Cation WPU (MEDA Mass ratio 13\%).

\begin{tabular}{ccccc}
\hline Raw material name & mol & $\mathbf{g}$ & Mass ratio \% & Quantity \\
\hline PTMG & 0.2 & 400 & 30.10 & 28.57 \\
3667 & 0.08 & 176 & 13.25 & 12.57 \\
IDPI & 2.1 & 466.81 & 35.13 & 33.34 \\
DBTL & & & 1 & \\
MDEA & 1.45 & 172.78 & 13 & 12.34 \\
KH550 & 0.45 & 99.63 & 7.5 & 6.46 \\
Bromabutane & 1.45 & 198.68 & & 7.12 \\
Acetic acid & & & & 14.81 \\
Butanediol & 0.15 & 13.52 & 1.02 & 0.97 \\
\hline
\end{tabular}


KH550 was added and mixed for 10 minutes and as a catalyst DBTDL was introduced. Then, acetic acid as a neutralizing agent was not added into the reaction until the mixture was slowly cooled to $40^{\circ} \mathrm{C}$. The neutralization reaction proceeded at the same temperature for $30 \mathrm{~min}$. When the synthesis of CWPU with MDEA volume ratio 13\% was done, 1,4-butanediol (BDO 1,4) was used as a different reagent.

\subsection{Preparation of Graphene-Based on Cellulose Nanocrystal}

Graphene solution was prepared with graphite oxide and cellulose nanocrystal to make at three types of ratio (graphite $=0.5 \mathrm{~g}):(\mathrm{CNC}=1 \mathrm{~g})$, (graphite $=1 \mathrm{~g}$ ): $(\mathrm{CNC}=1 \mathrm{~g})$, (graphite $=2 \mathrm{~g}):(\mathrm{CNC}=1 \mathrm{~g})$. The volume of solution was $100 \mathrm{ml}$ including graphite oxide, $\mathrm{CNC}$ and water and it was kept at cold water temperature for 90 minutes by mixing with stirring instrument and then the solution was held on centrifugal force instrument for removing residue of particles at speed of 4000 for 2 minutes.

\subsection{Application of Anti-Static Agent for Coating Foam on the Surface of Cashmere}

Firstly, required size of Cashmere fabric was cut and prepared to test for anti-static properties. In order to prepare the anti-static agent, each of three WPUs and graphene-CNC was weighted by ratio. For cationic waterborne polyurethanes, vesicant Sodium dodecyl sulfate (K12) which is to make the solution to become a bubble, graphene-CNC solution that was made in advance, sodium alginate and water at the bulk of $50 \mathrm{ml}$ were used, while for anionic waterborne polyurethane, the anti-static solution was made of AWPU and further chemicals. Ready anti-static agents and fabric were tested by FP020 Foam system (Shanghai Yuhui Chemical Co., Ltd.) and results were recorded.

\subsection{Characterization of WPUs, Graphene and Anti-Static Property of Cashmere}

1) A latex film was prepared for further testing by casting the emulsion on a Teflon plate and drying at ambient temperature for 7 days and then in a vacuum system at $50^{\circ} \mathrm{C}$ for 2 days to characterize three forms of waterborne polyurethanes such as two cationic and anionic polyurethanes by three characterizations such as film resistance to water as well as film resistance to Acid. Characterizations of three types of WPUs are film resistance to water and film resistance to acid. These two tests were examined during the 3 days which are from an initial to the final time. The results are illustrated in Table 3 and Table 4. Polyurethane and two forms of cationic waterborne polyurethanes which for film resistances to water and acid were found as expected.

2) The graphite and graphene-cellulose nanocrystal was tested by three analyzers of Nicolet iS5 Fourier Transform Infrared spectrometer (FTIR Thermo Scientific Company USA), TGA and XDR. The FTIR spectra infrared tester was 
Table 3. Film resistance to water.

\begin{tabular}{cccccc}
\hline Time & PU $3667+2348$ & PU 3030 & PUA 2348 & PUA 3667 + 2348 & PUA 3030 \\
\hline 0 hour & 0.090 & 0.130 & 0.180 & 0.210 & 0.170 \\
3 hour & 0.130 & 0.160 & 0.160 & 0.240 & 0.130 \\
8 hour & 0.102 & 0.143 & 0.168 & 0.322 & 0.272 \\
1 day & 0.110 & 0.145 & 0.179 & 0.334 & 0.267 \\
2 day & 0.115 & 0.146 & 0.175 & 0.327 & 0.259 \\
3 day & 0.113 & 0.144 & 0.174 & 0.327 & 0.260 \\
\hline
\end{tabular}

Table 4. Film resistance to acid.

\begin{tabular}{ccccccc}
\hline Time & PU 2348 & PU 3667 +2348 & PU 3030 & PUA 2348 & PUA $3667+2348$ & PUA 3030 \\
\hline 0 hour & 0.169 & 0.124 & 0.161 & 0.109 & 0.180 & 0.168 \\
3 hour & 0.231 & 0.160 & 0.180 & 0.153 & 0.240 & 0.210 \\
$\mathbf{8}$ hour & 0.228 & 0.163 & 0.187 & 0.156 & 0.249 & 0.226 \\
$\mathbf{1}$ day & 0.220 & 0.170 & 0.200 & 0.160 & 0.260 & 0.240 \\
2 day & 0.221 & 0.182 & 0.202 & 0.165 & 0.264 & 0.238 \\
3 day & 0.225 & 0.197 & 0.203 & 0.174 & 0.268 & 0.235 \\
\hline
\end{tabular}

used to analyze sample at the resolution of $3 \mathrm{~cm}^{-1}$, scanning from $4000 \mathrm{~cm}^{-1}$ to $500 \mathrm{~cm}^{-1}$. The analysis was carried out at ambient temperature.

3) The anti-static property of Cashmere fabric was tested by FY342E-II Fabric inductive electrostatic meter. Before testing samples, the each of samples was prepared through coating anti-static agents with foam method on the surface of the fabric and the coated samples were dried by Vacuum Drying Chamber (Shanghaijinghong Co., Ltd.). Results taken by Fabric inductive electrostatic meter were recorded. After testing samples for an electrostatic property, samples were observed to check how the graphene-CNC fixed on the surface of Cashmere fabric by S-4800 Emission Scanning Electron Microscope (Hitachi Japan) and figures were recorded and presented as good results.

\section{Results and Discussions}

The FTIR analysis: The FTIR spectra test was conducted to determine the chemical compositions on Graphite, Cellulose Nano-Crystal and three forms of the Graphene by three ratios ssuch as (graphite $=0.5)$ : $(\mathrm{CNC}=1)$; (graphite $=$ 1): $(\mathrm{CNC}=1)$; (graphite $=2)$ : $(\mathrm{CNC}=1)$. The result of the CNC taken by FTIR spectra shows that The FT-IR spectra for cellulose nanocrystal, CNC is shown in Figure 1; the peak at $1605 \mathrm{~cm}^{-1}$ corresponds to $\mathrm{C}=\mathrm{C}$ stretching vibrations of CNC. The cellulose nanocrystal illustrates $\mathrm{OH}$ stretching $3400 \mathrm{~cm}^{-1}$ and $\mathrm{C}-\mathrm{H}$ stretching $2850 \mathrm{~cm}^{-1}$ corresponding to the aliphatic moieties. The strong absorption peak at $1058 \mathrm{~cm}^{-1}$ ought to be the stretching vibration of the $\mathrm{C}-\mathrm{O}-\mathrm{C}$ bond in cellulose nanocrystal. $\mathrm{C}-\mathrm{O}$ and $\mathrm{C}=\mathrm{O}$ bond of Cellulose hit $1058 \mathrm{~cm}^{-1}$ and $1725 \mathrm{~cm}^{-1}$ 


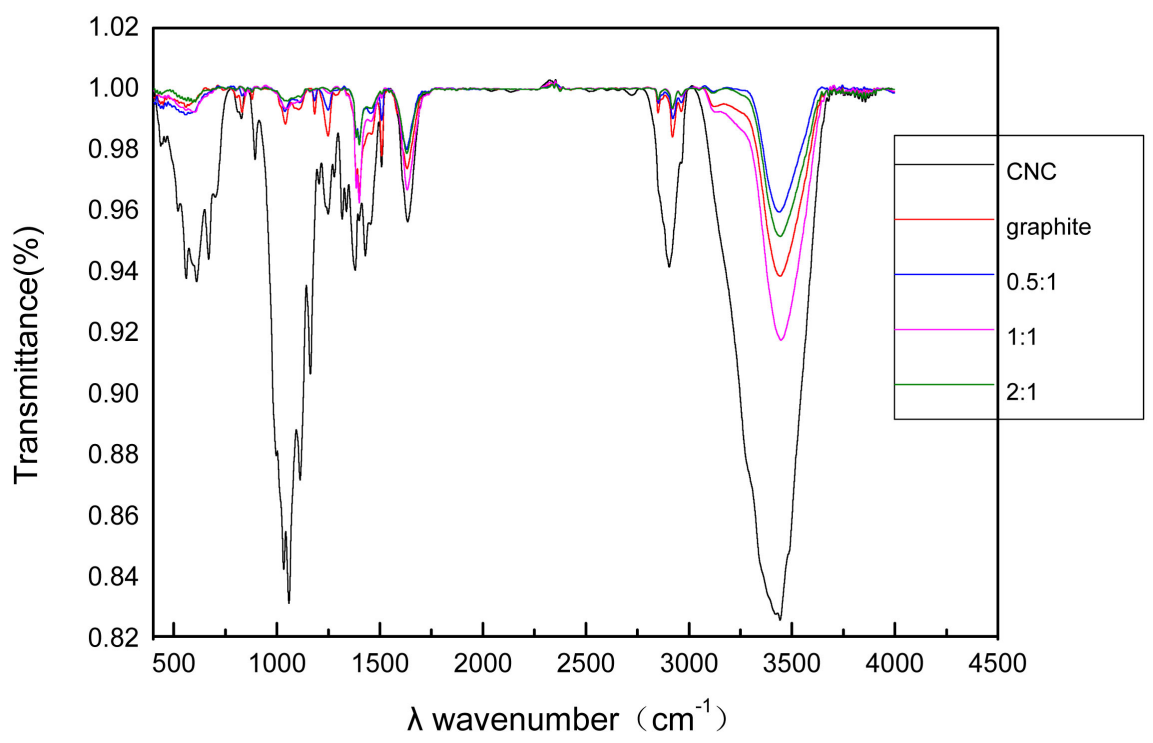

Figure 1. The FTIR spectra of CNC, Graphite and Graphene $(0.5: 1 ; 1: 1 ; 2: 1)$.

as it is shown in Figure 1. These characteristic peaks show on CNC that Cellulose nanocrystal was truly introduced in the Graphite to prepare Graphene. It can be seen from the figure that the absorption peak of about $3400 \mathrm{~cm}^{-1}$ belongs to the stretching vibration of $-\mathrm{OH}$, while the surface of $\mathrm{CNC}$ without graphene modification contains a large number of hydroxyl groups, which makes it difficult to disperse uniformly in ordinary solvents, and the amount of CNC hydrox$\mathrm{yl}$ groups after modification decreases, indicating that the dispersion of CNC in water can be improved.

Generally, the FTIR signals of Graphite was identified as a following; C-Cl signal bonds arranged around $600 \mathrm{~cm}^{-1}$, aromatic ring $\mathrm{C}=\mathrm{C}$ bonds showed at peak 1600 . The peak of absorbance was $2750 \mathrm{~cm}^{-1}$ and $1650 \mathrm{~cm}^{-1}$ signal is due to internal $\mathrm{CH}$ respectively. The strong and long signals indicating that they are more in particular bonds in tat regions or radiations. Because the mass of the atom increases, usually the wavenumber will decrease. As shown in Figure 1. When it is at aromatic signal 1600 which is due to beneze ring the conguregation goes up. These upwards and downwards were observed on graphene forms. As an example when compared to graphene $0.5: 1$ ratio with the graphenes $1: 1 ; 2: 1 \mathrm{ra}$ tio, it can be contemplated that the absorbance percentages rose up substantially in accordance with increasing of ratios of the graphene which means that the amount of light energy that passes through the samples increased. As an example, $\mathrm{OH}, \mathrm{C}=\mathrm{O}$ and $\mathrm{C}-\mathrm{O}$ bonds that made up around the peak of $3400 \mathrm{~cm}^{-1}, 1780$ $\mathrm{cm}^{-1}$ and $1087 \mathrm{~cm}^{-1}$ on graphene $0.5: 1$ ratio comprised $0.96 \%, 0.98 \%$ and $0.99 \%$ of transmittance while that transmittance percent was $0.92 \%, 0.97$ and 0.98 at the same peak of $3400 \mathrm{~cm}^{-1}$ on graphene 1:1 ratio. The identical parameters were recorded with other chemical compounds when graphene 2:1 ratio was compared with two ratios $(0.5: 1$ and $1: 1)$.

The XRD analysis: While testing samples of Cellulose nanocrystal (CNC), graphite as well as three forms of graphene by ratios (graphite $=0.5)$ : $(\mathrm{CNC}=1)$; 
(graphite $=1)$ : $(\mathrm{CNC}=1)$; (graphite $=2)$ : $(\mathrm{CNC}=1)$ by X-ray diffraction $(\mathrm{XRD})$ analyzer, the following results were obtained and shown in Figure 2. The peak at 21.7 degrees was elucidated as relatively weak regarding the crystallinity, according to the line of cellulose nanocrystal (CNC). When reviewed three samples of Graphene such as (graphite $=0.5)$ : $(\mathrm{CNC}=1)$; (graphite $=1)$ : $(\mathrm{CNC}=1)$; (graphite $=2)$ : $(\mathrm{CNC}=1)$, three different the points were recorded 25.5 degrees. It can be seen that graphite has a strong diffraction peak at 26.8 degrees and the peak is high and sharp which indicates the crystallinity of this sample is very high. The intensity of diffraction peak goes down slightly with the increase of $\mathrm{CNC}$ ratio which indicates that the more $\mathrm{CNC}$ content, the greater the degree of peeling of graphite powder, thus reducing the degree of crystallization of graphite powder was shown from the three lines of graphene' samples which were presented in Figure 2.

The TGA analysis: When Thermalgravimetric analysis (TGA) was carried out, the following indications which were shown in Figure 3 were gained. They are explained as a following:

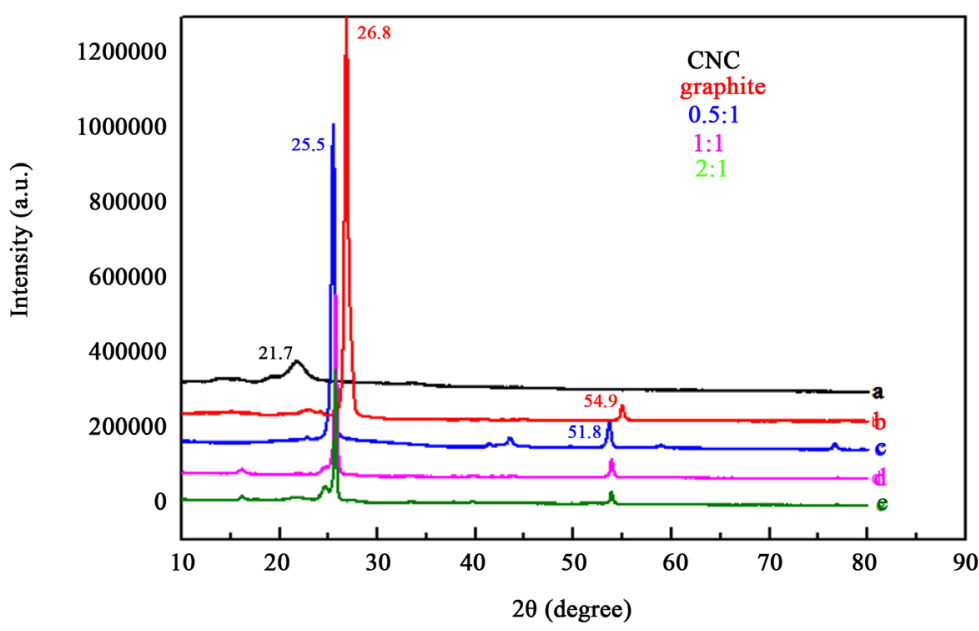

Figure 2. X-ray diffraction of CNC, graphite, graphene (0.5:1, 1:1, 2:1).

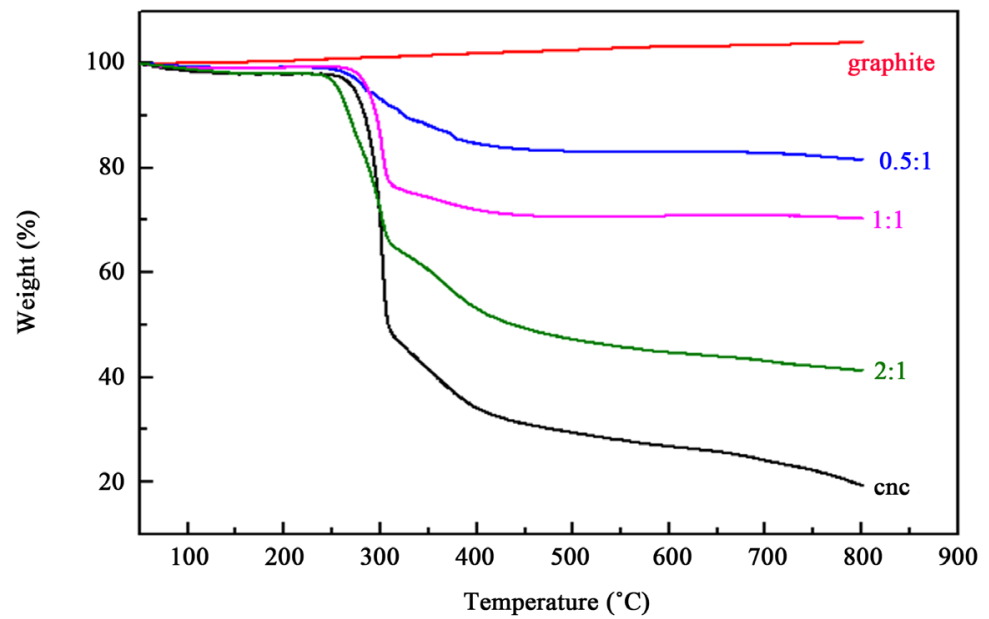

Figure 3. TGA of CNC, graphite and graphene (0.5:1, 1:1, 2:1). 
If the curve of graphite is examined to identify the indices relating to $\mathrm{Y}$-axis (weight \%) and $\mathrm{X}$-axis (Temperature ${ }^{\circ} \mathrm{C}$ ), it can be understood that when the temperature hits $800^{\circ} \mathrm{C}$, losing the mass on the pure graphite powder is not observed. Because of inaccurate baseline and inaccurate instrument precision, the mere upward trend may occur.

According to the curve of cellulose nanocrystal (CNC), the curve can be shown that due to the surface of Cellulose nanocrystal (CNC) which contains a great share of hydroxyl groups, there was a drammatic increase by $80.7 \%$ in the weight loss rate, when the temperature reached as same as the temperature of the pure graphite powder.

The weightlessness rates of $\mathrm{CNC/graphite} \mathrm{powder} \mathrm{solutions} \mathrm{with} \mathrm{three} \mathrm{pro-}$ portions of $0.5: 1,1: 1$, and $2: 1$ were $18.4 \%, 29.6 \%$ and $58.8 \%$ respectively, indicating that with the increase of CNC dosage, the more hydroxyl groups were added, and the weightlessness rates increased with the increase of CNC concentration.

The SEM analysis: The Scanning electron microscopy shows the images of Cashmere fabric which was treated by grapheme $(0.5: 1,1: 1,2: 1)$ in Figure 4 . The microscopic images were taken by the magnification of $\times 2500$ and $5 \mathrm{kV}, 30 \mu \mathrm{m}$. it is clear that when the images of untreated Cashmere fabric are observed, it can be that Cashmere fabric has roughness on its surface before coating the graphene. While the samples of GO which were made of three different ratios of Graphite were coating by the foam method, the surface roughness of Cashmere fabric dispersed as shown the images. Additionally, the sample of fabric coated with graphene 2:1 ratio showed a better film on the surface than the other two samples of Cashmere and anti-static properties as well. It can be assumed that when the surface has less roughness and if the graphene film occupies well on the surface of the fabric, the Fabric can be provided with the optimum improvements such as anti-static as we expected. As an example, Figures 1-4 were presented as a following.

\section{Conclusions}

The results of the analysis we have taken lead to the following conclusion. It was observed that the graphene can be fixed to the surface of fabric well by introducing waterborne polyurethanes (WPU) and it is not easy to fall off. When compared with the foam treated fabric, the direct coating can save more raw materials and the coating is more uniform.

The fabrics treated with graphene show good antistatic and the concentration of graphene will affect the conductivity. The higher the concentration makes the conductivity better. The CNC is more used to peel graphite powder, the better the peeling effect and the better the graphene performance. However, with the increase of CNC intercalation, the conductivity of graphene will be affected to some extent. Due to the low concentration of graphene and poor moisture absorption of fabrics, some raw materials will be lost during the foam treatment process. Therefore, it is hoped that a drying device can be installed below the 

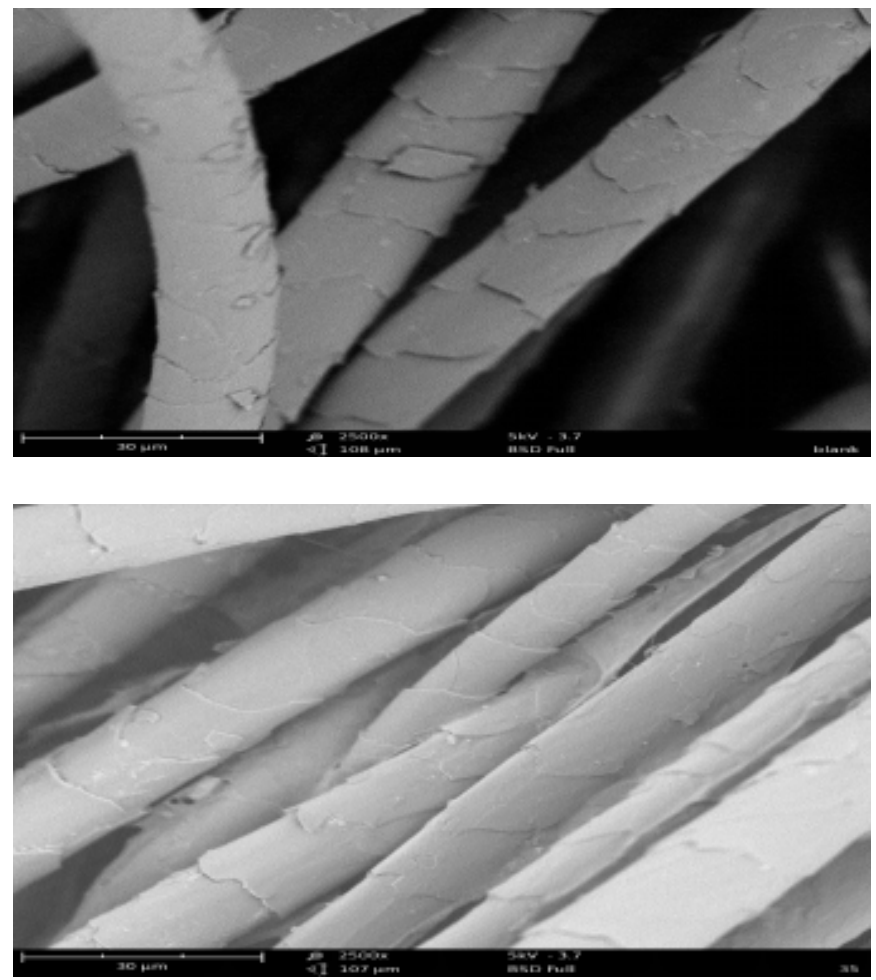

Untreated Cashmere fabric Cashmere coated with graphene (graphite $=0.5: \mathrm{CNC}=1$ )
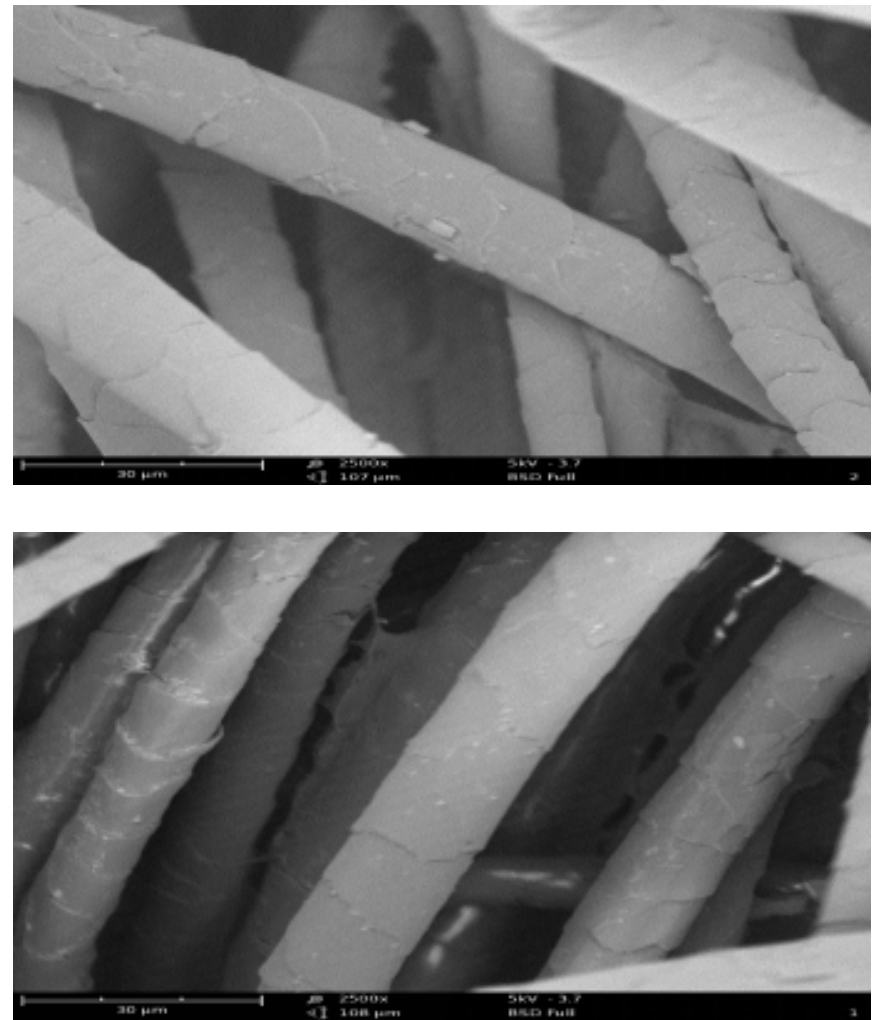

Cashmere coated with grapheme (graphite $=1: \mathrm{CNC}=1$ ) Cashmere coated with graphene (graphite $=2: \mathrm{CNC}=1$ )

Figure 4. The scanning electron microscopy images of Cashmere fabric coated with grapheme. 
coating machine to directly evaporate the water in the solution so that the graphene can be coated on the fabric 100 percent.

\section{Conflicts of Interest}

The authors declare no conflicts of interest regarding the publication of this paper.

\section{References}

[1] Li, M., Liu, F., Li, Y. and Qiang, X. (2017) Synthesis of Stable Cationic Waterborne Polyurethane with a High Solid Content: Insight from Simulation to Experiment. RSC Advances, 7, 13312. https://doi.org/10.1039/C7RA00647K

[2] Wang, H.-H. and Gen, C.-T. (2002) Synthesis of Anionic Water-Borne Polyurethane with the Covalent Bond of a Reactive Dye. Journal of Applied Polymer Science, 84, 797-805. https://doi.org/10.1002/app.10336

[3] Dong, H.J., Kim, E.Y., Kang, Y.S. and Kim, B.K. (2010) High Solid and High Performance UV Cured Waterborne Polyurethanes. Colloids and Surfaces A, 370, 58-63. https://doi.org/10.1016/j.colsurfa.2010.08.046

[4] Peng, S.J., Jin, Y., Cheng, X.F., Sun, T.B., Qi, R. and Fan, B.Z. (2015) A New Method to Synthesize High Solid Content Waterborne Polyurethanes by Strict Control of Bimodal Particle Size Distribution. Progress in Organic Coatings, 86, 1-10. https://doi.org/10.1016/j.porgcoat.2015.03.013

[5] Hou, L., Ding, Y., Zhang, Z., Sun, Z. and Shan, Z. (2015) Synergistic Effect of Anionic and Nonionic Monomers on the Synthesis of High Solid Content Waterborne Polyurethane. Colloids and Surfaces A, 467, 46-56.

[6] Burja, K., Segedin, U., Skale, S., Berce, P., Sket, P., Prosen, P. and Kukanja, D. (2015) Improved Anticorrosion Properties of Polyurethane Coatings Based on High-Solids Acrylics Synthesized in a High Pressure Reactor. Progress in Organic Coatings, 78, 275-286. https://doi.org/10.1016/j.porgcoat.2014.08.008

[7] Peng, S.J., Jin, Y., Sun, T., Qi, R., Fan, B.Z. and Cheng, X.F. (2014) Synthesis of High Solid Content Waterborne Polyurethanes with Controllable Bimodal Particle Size Distribution. Journal of Applied Polymer Science, 131, Article ID: 40420. https://doi.org/10.1002/app.40420

[8] Zhang, M., Hemp, S.T., Zhang, M., Allen, M.H., Carmean, R.N., Moore, R.B. and Long, T.E. (2015) Water-Dispersible Cationic Polyurethanes Containing Pendant Trialkylphosphoniums. Polymer Chemistry, 5, 3795-3803. https://doi.org/10.1039/C3PY01779F

[9] Botha, A.F. and Roux, J.A. (2008) The Fibre, Yarn and Fabric Properties of the Cashmere Component of South African Indigenous Goat Hair. CSIR Materials Science and Manufacturing.

[10] Zanini, S., Attilio Citterio, E.G. and Riccardi, C. (2015) Characterization of Atmospheric Pressure Plasma Treated Pure Cashmere and Wool/Cashmere Textiles: Treatment in Air/Water Vapor Mixture. Applied Surface Science, 349, 235-240. https://doi.org/10.1016/j.apsusc.2015.05.010

[11] Žilinskas, P.J., Lozovski, T., Jankauskas, V., Jurkšus, J. (2013) Electrostatic Properties and Characterization of Textile Materials Affected by Ion Flux. Materials Science, 19, No. 1. https://doi.org/10.5755/j01.ms.19.1.3828

[12] Zhang, C., Zhang, R.Z., Ma, Y.Q., Guan, W.B., Wu, X.L., Liu, X., Li, H., Du, Y.L. and Pan, C.P. (2014) Preparation of Cellulose/Graphene Composite and Its Appli- 
cations for Triazine Pesticides Adsorption from Water. ACS Sustainable Chemistry \& Engineering, 10, 14.

[13] Montes, S., Etxeberria, A., Mocholi, V., Rekondo, A., Grande, H. and Labidi, J. (2018) Effect of Combining Cellulose Nanocrystals and Graphenenanoplatelets on the Properties of Poly(Lactic Acid) Based Films. eXPRESS Polymer Letters, 12, 543-555.

[14] Valentini, L., Bittolo Bon, S., Fortunat, E. and Kenny, J.M. (2014) Preparation of Transparent and Conductive Cellulose Nanocrystals/Graphenenanoplatelets Films. Journal of Materials Science, 49, 1009-1013. https://doi.org/10.1007/s10853-013-7776-9

[15] Valentini, L., et al. (2013) A Novel Method to Prepare Conductive Nanocrystalline Cellulose/Graphene Oxide Composite Films. Materials Letters, 105, 4-7. https://doi.org/10.1016/j.matlet.2013.04.034

[16] Lv, J.L., Zhang, G.Q., Zhang, H.M. and Yang, F.L. (2018) Graphene Oxide-Cellulose Nanocrystal (GO-CNC) Composite Functionalized PVDF Membrane with Improved Antifouling Performance in MBR: Behavior and Mechanism. Chemical Engineering Journal, 352, 765-773. https://doi.org/10.1016/j.cej.2018.07.088

[17] Pottathara, Y.B. (2017) Graphene Based Composites with Cellulose Nanofibrils for Energy Storage Applications. Textile Research Journal, 49, 156.

[18] Namboodri, C.G. and Duke, M.W. (1979) Foam Finishing of Cotton-Containing Textiles. Textile Research Journal, 49, 156-162.

https://doi.org/10.1177/004051757904900308 